PACIFIC JOURNAL OF MATHEMATICS

Vol. 178, No. 1, 1997

\title{
PERMUTABILITY OF CHARACTERS ON ALGEBRAS
}

\author{
L. Grunenfelder, R. Guralnick, T. Košir and H. Radjavi
}

An algebra of matrices $\mathcal{A}$ with Jacobson radical $\mathcal{R}$ is said to have permutable trace if $\operatorname{Tr}(a b c)=\operatorname{Tr}(b a c)$ for all $a, b, c$ in $\mathcal{A}$. We show in this paper that in characteristic zero $\mathcal{A}$ has permutable trace if and only if $\mathcal{A} / \mathcal{R}$ is commutative. Generalizing to arbitrary characteristic we find that the result still holds when the trace form of $\mathcal{A}$ is non-degenerate. Finally, in positive characteristic, slightly stronger condition of permutability of the Brauer character is shown to be equivalent to the commutativity of $\mathcal{A} / \mathcal{R}$.

\section{Introduction.}

First consider an algebra $\mathcal{A}$ of $n \times n$ matrices over a field $F$. We say that $\mathcal{A}$ has permutable trace if the traces $\operatorname{Tr}(a b c)$ and $\operatorname{Tr}(b a c)$ are equal for any matrices $a, b, c \in \mathcal{A}$. If the characteristic of $F$ is zero then our main result implies that $\mathcal{A}$ has permutable trace if and only if $\mathcal{A} / \mathcal{R}$ is commutative, where $\mathcal{R}$ is the (Jacobson) radical of $\mathcal{A}$. In particular, it follows that a division algebra (or a semisimple algebra) is commutative if and only if it has permutable trace. If the characteristic of $F$ is positive then the result is valid when the size of the matrices is small, i.e. if $n<2 p$.

In Section 3 we investigate the obstructions when $n \geq 2 p$ by considering properties of the trace form on $\mathcal{A}$, defined by $(a, b)=\operatorname{Tr}(a b)$. This is a symmetric, associative, bilinear form on $\mathcal{A}$. In particular, whenever this trace form on $\mathcal{A}$ is non-degenerate, then in any characteristic, $\mathcal{A}$ has permutable trace if and only if $\mathcal{A} / \mathcal{R}$ is commutative.

These considerations suggest a slightly stronger condition than permutability of the trace to force commutativity of $\mathcal{A} / \mathcal{R}$. In Section 4 we show that if the trace is replaced by the Brauer character, suitably generalized as in [3], then the original result is restored in full generality, i.e. $\mathcal{A} / \mathcal{R}$ is commutative if and only if the Brauer character is permutable.

A version of our main theorem for an algebra of matrices over an algebraically closed field of characteristic zero was proved in $[\mathbf{5}, \mathbf{6}]$. 


\section{Algebras with permutable trace.}

For any finite-dimensional representation $\rho: \mathcal{A} \rightarrow \operatorname{End}_{F}(V)$ let $t_{V}: \mathcal{A} \rightarrow F$ be the associated character or trace, i.e. $t_{V}(a)=\operatorname{Tr}(\rho a)$. If the representation is faithful, we say that the character is faithful. The character $t_{V}: \mathcal{A} \rightarrow F$ is called permutable if $t_{V}(a b c)=t_{V}(b a c)$. This is equivalent to saying that $t_{V}\left(a_{1} a_{2} \ldots a_{k}\right)=t_{V}\left(a_{\sigma(1)} a_{\sigma(2)} \ldots a_{\sigma(k)}\right)$ for all $k>0$, any $a_{1}, a_{2}, \ldots, a_{k} \in \mathcal{A}$ and every permutation $\sigma$ of $\{1,2, \ldots, k\}$.

More generally $\left[4\right.$, Ch. 16], the characteristic polynomial $p_{V}: \mathcal{A} \rightarrow F[x]$ of the representation $\rho: \mathcal{A} \rightarrow \operatorname{End}_{F}(V)$, defined by $p_{V}(a)=\operatorname{det}(x I-$ $\rho(a))$, satisfies $p_{V}(a b)=p_{V}(b a)$. We say that $p_{V}$ is permutable if $p_{V}(a b c)=$ $p_{V}(b a c)$.

Every field extension $K / F$ induces a representation $\rho_{K}=\rho \otimes_{F} K: \mathcal{A} \otimes_{F}$ $K \rightarrow \operatorname{End}(V) \otimes_{F} K \cong \operatorname{End}\left(V \otimes_{F} K\right)$ of the $K$-algebra $\mathcal{A}_{K}=\mathcal{A} \otimes_{F} K$ in the vector space $V_{K}=V \otimes_{F} K$. The $\mathcal{A}$-module $V$ has a composition series

$$
0=V_{r+1} \subset V_{r} \subset \ldots \subset V_{2} \subset V_{1}=V
$$

with simple factors $W_{j}=V_{j} / V_{j+1}$. The $\mathcal{A}$-module $W=\oplus_{j=1}^{r} W_{j}$ is semisimple, it is called the semisimple module associated with $V$. If $\mathcal{I}_{W}$ is the annihilator ideal of $W$ in $\mathcal{A}$ then $\mathcal{I}_{W} V_{j} \subset V_{j+1}$ for $j \geq 1$, and so $\mathcal{I}_{W}^{r+1} \subseteq \mathcal{I}_{V}$. In particular, if $V$ is a faithful $\mathcal{A}$-module then $\mathcal{I}_{W}$ is nilpotent. (We refer to $[\mathbf{1}, 4]$ for details.)

In the proofs we will often replace the direct sum $\oplus_{j=1}^{r} W_{j}$ with the direct sum $\oplus_{i=1}^{s} W_{i}^{m_{i}}$, where $W_{i}$ are the non-isomorphic composition factors of $V$.

Lemma 2.1. Let $\mathcal{A}$ be a finite dimensional $F$-algebra, $V$ a finite dimensional $\mathcal{A}$-module, $W=\oplus_{i=1}^{s} W_{i}^{m_{i}}$ the associated semisimple $\mathcal{A}$-module and let $K / F$ be a field extension. Then,

(1) $\mathcal{A} / \mathcal{R}$ is commutative if and only if $\mathcal{A}_{K} / \mathcal{R}\left(\mathcal{A}_{K}\right)$ is commutative;

(2) $t_{V}$ is permutable if and only if $t_{V_{K}}$ is permutable;

(3) $t_{V}=t_{W}=\sum_{i=1}^{s} m_{i} t_{W_{i}}$;

(4) $p_{V}=p_{W}=\prod_{i=1}^{s} p_{W_{i}}^{m_{i}}$.

Proof. Since the radical of a finite dimensional algebra is the maximal nilpotent ideal it clearly follows that $\mathcal{R}(\mathcal{A})_{K} \subseteq \mathcal{R}\left(\mathcal{A}_{K}\right)$. If $\mathcal{A} / \mathcal{R}$ is commutative then $\mathcal{A}_{K} / \mathcal{R}_{K} \cong \mathcal{A} / \mathcal{R} \otimes_{F} K$ is commutative and hence so is $\mathcal{A}_{K} / \mathcal{R}\left(\mathcal{A}_{K}\right)$. Conversely, suppose that $\mathcal{A}_{K} / \mathcal{R}\left(\mathcal{A}_{K}\right)$ is commutative. Then $(a b-b a) \otimes_{F} 1 \in$ $\mathcal{R}\left(\mathcal{A}_{K}\right)$ for any two elements $a$ and $b$ of $\mathcal{A}$. If $\mathcal{I}$ is the ideal of $\mathcal{A}$ generated by $a b-b a$ then the ideal $\mathcal{I}_{K}$ of $\mathcal{A}_{K}$ is contained in $\mathcal{R}\left(\mathcal{A}_{K}\right)$ and is therefore nilpotent. But then $\mathcal{I}$ is nilpotent and so is contained in $\mathcal{R}$. The second assertion follows directly from the fact that $t_{V_{K}}\left(a \otimes_{F} k\right)=t_{V}(a) k$. To prove 
the last two assertions it suffices to show that $t_{V}=t_{V^{\prime}}+t_{V^{\prime \prime}}$ and $p_{V}=p_{V^{\prime}} p_{V^{\prime \prime}}$ for every exact sequence of finite dimensional $\mathcal{A}$-modules

$$
0 \rightarrow V^{\prime} \rightarrow V \stackrel{\eta}{\rightarrow} V^{\prime \prime} \rightarrow 0
$$

But, if $\left\{e_{i} \mid i=1,2, \ldots, m\right\}$ is a basis of $V$ with dual basis $\left\{e^{i} \mid i=1,2, \ldots, m\right\}$ such that $\left\{e_{j} \mid j=1,2, \ldots r\right\}$ is a basis of $V^{\prime}$ and $\left\{f_{j}=\eta\left(e_{j}\right) \mid j=r+1, r+\right.$ $2, \ldots, m\}$ a basis of $V^{\prime \prime}$ then

$$
t_{V}(a)=\sum_{i=1}^{m} e^{i}\left(a e_{i}\right)=\sum_{j=1}^{r} e^{j}\left(a e_{j}\right)+\sum_{j=r+1}^{m} f^{j}\left(a f_{j}\right)=t_{V^{\prime}}(a)+t_{V^{\prime \prime}}(a) .
$$

Moreover, since $e^{i}\left(a e_{j}\right)=0$ when $i>r$ and $j<r$, and $e^{i}\left(a e_{j}\right)=f^{i}\left(a f_{j}\right)$ if $i, j>r$ we see that $p_{V}=p_{V^{\prime}} p_{V^{\prime \prime}}$. The result now follows by induction.

Theorem 2.2. Suppose that either char $F=0$ or char $F=p>0$ and $n<2 p$. Then a subalgebra $\mathcal{A}$ of $M_{n}(F) \cong \operatorname{End}_{F}(V)$ has permutable trace if and only if $\mathcal{A} / \mathcal{R}$ is commutative. In particular, a semisimple algebra has permutable trace if and only if it is commutative.

Proof. By Lemma 2.1, both properties, that of permutable trace and of the commutativity of $\mathcal{A} / \mathcal{R}$, are invariant under field extensions. So we may assume that $F$ is algebraically closed. By the third part of Lemma 2.1 we also may replace $V$ by the semisimple $\mathcal{A}$-module $W=\oplus_{i=1}^{s} W_{i}^{m_{i}}$, where the $W_{i}$ are the non-isomorphic simple composition factors of $V$. But then $\mathcal{R} W=0$ and, since $V$ is a faithful $\mathcal{A}$-module, the annihilator $\mathcal{I}_{W}$ is nilpotent, so that $\mathcal{R}=\mathcal{I}_{W}$. This shows that we may assume that $\mathcal{A}$ is a semisimple algebra. So it suffices to prove the assertion of the theorem when $F$ is algebraically closed and $\mathcal{A}$ is a semisimple $F$-algebra. But then $\mathcal{A} \cong \oplus_{i=1}^{s} \mathcal{A}_{i}$, where $\mathcal{A}_{i} \cong M_{n_{i}}(F)$, and $\mathcal{A}$ is not commutative if and only if at least one of the $\mathcal{A}_{i}$ is not commutative, i.e. if and only if there is a central idempotent $E \in \mathcal{A}$ such that $E \mathcal{A}$ is a non-commutative simple $F$-algebra. Then $\mathcal{A}=E \mathcal{A} \oplus(1-E) \mathcal{A}$ and $V=E V \oplus(1-E) V$. So clearly, we may assume that $\mathcal{A}$ is a simple $F$-algebra. Then $\mathcal{A} \cong M_{d}(F)$ and $V \cong U^{m}$ is a direct sum of $m$ copies of the unique (up to isomorphism) simple $\mathcal{A}$-module, so that $t_{V}=m t_{U}$ and $t_{U}$ is just the ordinary trace on $M_{d}(F)$. If the characteristic of $F$ does not divide $m$ then $t_{V}$ is permutable if and only $t_{U}$ is. But the ordinary trace on $M_{d}(F)$ is permutable if and only if $d=1$, since $E_{11} E_{12} E_{21}=E_{11}$ and $E_{12} E_{11} E_{21}=0$. This completes the proof when the charateristic of $F$ is zero. When char $F=p>0$ we see that the assumptions that $p$ divides $m$ and $d>1$ imply that $2 p \leq m d \leq n$, and the assertion follows. 
Corollary 2.3. A finite dimensional simple $F$-subalgebra of $M_{n}(F)$, with $n<2 p$ if $F$ has positive characteristic $p$, has a permutable trace if and only if it is a finite field extension of $F$.

Corollary 2.4. Suppose that either $\operatorname{char} F=0$ or char $F=p>0$ and $n<2 p$. Then the following are equivalent for a subalgebra $\mathcal{A}$ of $M_{n}(F)$.

(1) $\mathcal{A}$ is triangularizable over some finite extension field $K / F$.

(2) $\mathcal{A}$ has permutable characteristic polynomial.

(3) $\mathcal{A}$ has permutable trace.

Remark 2.5. If char $F=p>0$ then the condition $n<2 p$ in Theorem 2.2 cannot be relaxed. For example, if $n=2 p$ and $\mathcal{A}$ is the image of the diagonal map $\Delta: M_{2}(F) \rightarrow M_{2}(F)^{p} \subset M_{n}(F)$, then $\operatorname{Tr}(a)=0$ for all $a \in \mathcal{A}$. In this case $\mathcal{A} / \mathcal{R} \cong M_{2}(F)$ is simple, but $W$ is the direct sum of $p$ copies of the unique simple $M_{2}(F)$-module $F^{2}$. So, it follows that $\mathcal{A}$ has permutable trace, but $\mathcal{A} / \mathcal{R} \cong M_{2}(F)$ is not commutative.

If $F$ is not perfect, then there are examples of a different nature. Let $K / F$ be a purely inseparable extension of degree $p$, let $\mathcal{A}=M_{2}(K)$ and let $V$ be the unique simple $\mathcal{A}$-module. Then $\operatorname{dim}_{K} V=2$ and $\operatorname{dim}_{F} V=2 p$, so that $\mathcal{A}$ imbeds in $M_{2 p}(F)$ giving a $2 p$-dimensional representation of the $F$-algebra $\mathcal{A}$. Since $t_{V / F}=\operatorname{Tr}_{K / F} \circ t_{V / K}$ (see [2, Sect. 22] or [7, Sect. 9]) and since the field trace $\operatorname{Tr}_{K / F}=0$ for any purely inseparable extension $K / F$ of positive degree we obtain $t_{V / F}=0$. Again, the trace of the faithful representation of $\mathcal{A}$ is permutable but the simple $F$-algebra $\mathcal{A}$ is not commutative.

We shall see in the next section that the only way permutability of the trace can hold without $\mathcal{A} / \mathcal{R}$ being commutative is that composition factors of the above two types occur.

\section{The trace form and permutability of trace.}

Let $\mathcal{A}$ be a subalgebra of $M_{n}(F) \cong \operatorname{End}_{F}(V)$ with Jacobson radical $\mathcal{R}$ and let $W_{1}, W_{2}, \ldots, W_{s}$ be the distinct composition factors with multiplicities $m_{1}, m_{2}, \ldots, m_{s}$ of the $\mathcal{A}$-module $V$. We have seen in Section 2 that $W=$ $\oplus_{i=1}^{s} W_{i}^{m_{i}}$ is a module over the semisimple algebra $\mathcal{A} / \mathcal{R}$ and that

$$
t_{V}=t_{W}=\sum_{i=1}^{s} m_{i} t_{W_{i}}
$$

This shows that for questions concerning permutability of trace one can essentially reduce to the semisimple case.

The bilinear form on $\mathcal{A} / \mathcal{R}$ associated with the trace $t_{V}: \mathcal{A} \rightarrow F$ is defined by $(a, b)=t_{V}(a b)$. This form is clearly symmetric and associative, 
i.e. $(a, b c)=(a b, c)$, so that its radical is an ideal. Observe that this trace form is non-degenerate if and only if $m_{i} t_{W_{i}}$ is non-zero for each $i$. This is because the radical of the trace form is an ideal, so that when $\mathcal{A}$ is simple the radical is either zero or the whole algebra $\mathcal{A}$. Sometimes we just say that the trace is non-degenerate if the associated trace form is non-degenerate.

Proposition 3.1. Let $\mathcal{A}=M_{n}(D)$ be a simple ring, where $D$ is a division algebra over the field $F$, and suppose that $V$ is a simple $\mathcal{A}$-module. Then $t_{V}=0$ if and only if either

(1) $Z(\mathcal{A}) / F$ is inseparable, or

(2) $[D: Z(\mathcal{A})]$ is a multiple of the characteristic.

Proof. By definition the degree of the division algebra $D$ over its center is $e$, where $e^{2}=[D: Z(D)]$. Now $t_{V / F}=\operatorname{Tr}_{Z(\mathcal{A}) / F} \circ t_{V / Z(\mathcal{A})}$ (see [2, Sect. 22] or [7, Sect. 9]), and the field trace $\operatorname{Tr}_{Z(\mathcal{A}) / F}$ is non-degenerate on $Z(\mathcal{A})$ if and only if $Z(\mathcal{A}) / F$ is separable and is zero otherwise. To compute the trace $t_{V / Z(\mathcal{A})}$ split $D$ by tensoring by a maximal subfield $K$ of $D$. Then $V \otimes_{Z} K \cong M^{e}$, where $M$ is the unique simple module over the simple $K$ algebra $\mathcal{A} \otimes_{Z} K \cong M_{e n}(K)$, and $t_{V / Z(\mathcal{A})}=e t_{M}$. Now the assertion follows, since $t_{M} \neq 0$.

Corollary 3.2. Let $\mathcal{A}$ be a subalgebra of $M_{n}(F) \cong \operatorname{End}_{F}(V)$. Then the trace $t_{V}$ is non-degenerate on $\mathcal{A} / \mathcal{R}$ if and only if all of the following conditions hold:

(1) $m_{i}$ is not divisible by the characteristic of $F$ for $i=1,2, \ldots, s$;

(2) $Z(\mathcal{A} / \mathcal{R})$ is separable over $F$;

(3) $[D: Z(D)]$ is not divisible by the characteristic of $F$ for every division algebra $D$ appearing in $\mathcal{A} / \mathcal{R}$.

These comments about the trace form allow us to extend our main result, Theorem 2.2, in several directions.

Theorem 3.3. Suppose that $\mathcal{A}$ is a matrix algebra with non-degenerate trace. Then, the trace is permutable if and only if $\mathcal{A} / \mathcal{R}$ is commutative.

If $F$ has characteristic zero, then all the conditions of the Corollary 3.2 are automatically satisfied, and so $t_{V}$ is always non-degenerate. Observe that passing to a separable extension a non-degenerate form remains nondegenerate, so one may reduce to the algebraically closed case whenever $F$ is perfect. If $F$ is perfect, then property (2) of Corollary 3.2 is obviously satisfied and by a result of Albert (see [3]) so is property (3).

Recall that, if $W$ is an $\mathcal{A}$-module, then $\mathcal{I}_{W}$ denotes the annihilator ideal of $W$ in $\mathcal{A}$. 
Theorem 3.4. Let $F$ be a perfect field and let $\mathcal{A}$ be a subalgebra of $M_{n}(F) \cong$ $\operatorname{End}_{F}(V)$. Then the trace $t_{V}$ is permutable if and only if each composition factor $W$ of $V$ for which $\mathcal{A} / \mathcal{I}_{W}$ is not commutative has multiplicity divisible by the characteristic of $F$.

\section{Algebras with permutable Brauer characters.}

An invariant for finite dimensional representations which, in positive characteristic, is finer than the ordinary character is the Brauer character. The more general version used here is described in [3]. As we have seen in Section 2 , there is no harm in replacing the field $F$ by its algebraic closure.

Let $F$ be a field of positive characteristic $p$ with algebraic closure $\bar{F}$. Then there exists an integral domain $D$ of characteristic zero satisfying the following conditions:

(1) There exists a surjection $f: D \rightarrow \bar{F}$; and

(2) $D$ is local, integrally closed and its quotient field $E$ is algebraically closed.

Here is the construction. Let $B$ be an integral domain of characteristic zero such that there exists a surjective homomorphism $f: B \rightarrow \bar{F}$. By laying over, we may replace $B$ by its integral closure in the algebraic closure of its quotient field. If $D$ is the localization of $B$ at the maximal ideal $\operatorname{ker} f$, then $D$ is a local integrally closed domain of characteristic zero with algebraically closed quotient field $E$ and a surjective homomorphism $f: D \rightarrow \bar{F}$.

Let $I=\operatorname{ker}(f)$. So $I$ is the unique maximal ideal of $D$. Then $f$ induces a surjective group homomorphism from $D^{*}$ to $\bar{F}^{*}$ with kernel $1+I$. Since $D$ is integrally closed and its quotient field is algebraically closed, it is straightforward to verify that $1+I$ is a divisible group. Thus, $f$ is a split surjection on $D^{*}$. Let $\mu: \bar{F}^{*} \rightarrow D^{*} \subset E^{*}$ be an injection with $f \mu(x)=x$ for all $x \in \bar{F}^{*}$. Define $\mu(0)=0$.

If $\rho: \mathcal{A} \rightarrow \operatorname{End}_{F}(V) \cong M_{n}(F)$ is a representation of the algebra $\mathcal{A}$, define the Brauer character $b_{V}: \mathcal{A} \rightarrow E$ by

$$
b_{V}(a)=\sum_{i=1}^{n} \mu\left(\lambda_{i}\right)
$$

where $\lambda_{1}, \lambda_{2}, \ldots, \lambda_{n}$ are the eigenvalues of $\rho(a)$ in $\bar{F}$. Observe that

$$
f\left(b_{V}(a)\right)=\sum_{i} f\left(\mu\left(\lambda_{i}\right)\right)=\operatorname{Tr}_{V}(a),
$$

so that the Brauer character contains at least as much information as the ordinary character. In fact [3], the Brauer character determines the composition factors of $\rho$ (while the trace in the algebraically closed case only 
determines the multiplicites of the composition factors modulo $p$ ). We can now obtain a version of Theorem 2.2 in positive characteristic by using the Brauer character. We first prove the analog of Lemma 2.1 for the Brauer character.

Lemma 4.1. Let $\mathcal{A}$ be a finite dimensional $F$-algebra, $V$ a finite dimensional $\mathcal{A}$-module, $W=\oplus_{i=1}^{s} W^{m_{i}}$ the associated semisimple $\mathcal{A}$-module and let $K / F$ be an field extension. Then,

(1) $\mathcal{A} / \mathcal{R}$ is commutative if and only if $\mathcal{A}_{K} / \mathcal{R}\left(\mathcal{A}_{K}\right)$ is commutative;

(2) $b_{V}$ is permutable if and only if $b_{V_{K}}$ is permutable;

(3) $b_{V}=b_{W}=\sum_{i=1}^{s} m_{i} b_{W_{i}}$.

Proof. The first assertion is the same as that in Lemma 2.1. The second assertion follows directly from the fact that $b_{V_{K}}\left(a \otimes_{F} k\right)=b_{V}(a) k$. For the third assertion we may assume that $F$ is algebraically closed and it suffices to show that $b_{V}=b_{V^{\prime}}+b_{V^{\prime \prime}}$ for every exact sequence of finite dimensional $\mathcal{A}$-modules

$$
0 \rightarrow V^{\prime} \rightarrow V \stackrel{\eta}{\rightarrow} V^{\prime \prime} \rightarrow 0
$$

Since $F$ is algebraically closed there is for every $a \in \mathcal{A}$ a basis $\left\{e_{i} \mid i=\right.$ $1,2, \ldots, r\}$ of $V^{\prime}$ in which $a$ is upper triangular, and this basis can be extended to a basis $\left\{e_{j} \mid j=1,2, \ldots n\right\}$ of $V$ in which $a$ is upper triangular. But then $\left\{\eta\left(e_{j}\right) \mid j=r+1, r+2, \ldots n\right\}$ is a basis of $V^{\prime \prime}$ in which $a$ is upper triangular. Then

$$
b_{V}(a)=\sum_{i=1}^{n} \lambda_{i}=\sum_{j=1}^{r} \lambda_{j}+\sum_{j=r+1}^{n} \lambda_{j}=b_{V^{\prime}}(a)+b_{V^{\prime \prime}}(a) .
$$

The result now follows by induction.

Using the Brauer character we now get the following refinements of the main results of Sections 2 and 3.

Theorem 4.2. $\quad A$ subalgebra $\mathcal{A}$ of $M_{n}(F) \cong \operatorname{End}_{F}(V)$ has permutable Brauer character if and only if $\mathcal{A} / \mathcal{R}$ is commutative.

Proof. Proceed along the same lines as in the proof of Theorem 2.2. Again, by Lemma 4.1, we see as in Theorem 2.2 that it is enough to prove the assertion when $F$ is algebraically closed and $\mathcal{A}$ is a semisimple $F$-algebra. But then $\mathcal{A} \cong \oplus_{i=1}^{s} \mathcal{A}_{i}$, where $\mathcal{A}_{i} \cong M_{n_{i}}(F)$, and $\mathcal{A}$ is not commutative if and only if at least one of the $\mathcal{A}_{i}$ is not commutative, i.e. if and only if there is a central idempotent $E \in \mathcal{A}$ such that $E \mathcal{A}$ is a non-commutative simple $F$-algebra. Then $\mathcal{A}=E \mathcal{A} \oplus(1-E) \mathcal{A}$ and $V=E V \oplus(1-E) V$. 
So, clearly, we may assume that $\mathcal{A}$ is a simple $F$-algebra. Then $\mathcal{A} \cong M_{d}(F)$ and $V \cong U^{m}$ is a direct sum of $m$ copies of the unique (up to isomorphism) simple $\mathcal{A}$-module, so that $b_{V}=m b_{U}$. It follows then that $b_{V}$ is permutable if and only if $b_{U}$ is. If $b_{U}$ is permutable, then so is $f \circ b_{U}$ which is the trace on $M_{d}(F)$. This trace is permutable if and only if $d=1$, since $E_{11} E_{12} E_{21}=E_{11}$ and $E_{12} E_{11} E_{21}=0$, and the proof is complete.

Corollary 4.3. The following are equivalent for an algebra $\mathcal{A}$ of matrices over the field $F$.

(1) $\mathcal{A}$ is triangularizable over some finite extension field $K / F$.

(2) $\mathcal{A}$ has permutable Brauer character.

(3) $\mathcal{A}$ has permutable characteristic polynomial.

Proof. (1) and (2) are equivalent by the theorem. Clearly, (1) implies (3). Since the characteristic polynomial determines the eigenvalues, whence the Brauer character, (3) implies (2).

\section{References}

[1] D.J. Benson, Representations and cohomology I, Cambridge University Press, 1991.

[2] P.K. Draxl, Skew Fields, Cambridge University Press, 1983.

[3] R.N. Gupta and R.M. Guralnick, Shirshov's Theorem and Representations of Semigroups, preprint.

[4] R.S. Pierce, Associative Algebras, Springer-Verlag, 1982.

[5] H. Radjavi, A Trace Condition Equivalent to Simultaneous Triangularizability, Can. J. Math., 38 (1986), 376-386.

[6] _ The Engel-Jacobson Theorem Revisited, J. Alg., 111 (1987), 427-430.

[7] I. Reiner, Maximal Orders, Academic Press, 1975.

Received June 10, 1995 and revised January 4, 1996.

DALHOUSIE UNIVERSITY

Halifax, Nova Scotia

B3H 3J5, CANADA

E-mail address: luzius@cs.dal.ca

UnIVERsity OF SOUTHERN CALIFORNIA

Los ANGEles, CA 90089-1113

UNIVERSITY OF LJUBLJANA

JADRANSKA 19

61000 Ljubluana, Slovenia

AND

DALHOUSIE UNIVERSITY

Halifax, Nova Scotia

B3H 3J5, CANADA 